\title{
White Organic Light-emitting Diode Using Nano-double Ultrathin Carrier-trapping Materials in Performance Stability
}

\author{
Juin J. Liou, ${ }^{1}$ Wen-Ray Chen, ${ }^{2 *}$ Chih-Chieh Kang, ${ }^{3}$ \\ Kuan-Wei Lee, ${ }^{4}$ Shih-Wei Feng, ${ }^{5}$ and Chien-Jung Huang ${ }^{5^{* *}}$ \\ ${ }^{1}$ School of Information Engineering, Zhengzhou University, \\ No. 100 Science Avenue, Zhengzhou, Henan Province, China \\ ${ }^{2}$ Department of Electronic Engineering, National Formosa University, \\ No. 64, Wunhua Rd., Huwei Township, Yunlin 632, Taiwan \\ ${ }^{3}$ Department of Electro-Optical Engineering, Southern Taiwan University of Science and Technology, \\ No. 1, Nan-Tai Street, Yungkang Dist., Tainan 710, Taiwan \\ ${ }^{4}$ Department of Electronic Engineering, I-Shou University, \\ No. 1, Sec. 1, Syuecheng Rd., Dashu Dist., Kaohsiung 84001, Taiwan \\ ${ }^{5}$ Department of Applied Physics, National University of Kaohsiung, \\ \#700, Kaohsiung University Rd., Nanzih Dist., Kaohsiung 811, Taiwan
}

(Received May 17, 2018; accepted August 3, 2018)

Keywords: trapping layer, electroluminescence, charge trapping, color purity, OLED

The structure of indium tin oxide (ITO) $(100 \mathrm{~nm}) /$ molybdenum trioxide $\left(\mathrm{MoO}_{3}\right)(15 \mathrm{~nm}) /$ N,N0-bis-(1-naphthyl)-N,N0-biphenyl-1,10-biphenyl-4,40-diamine (NPB) $(40 \mathrm{~nm}) / 4,4$ '-bis(2,2diphenylvinyl)-1,1'-biphenyl (DPVBi) $(10 \mathrm{~nm}) / 5,6,11,12$-tetraphenylnaphthacene (rubrene) $(0.2 \mathrm{~nm}) /$ DPVBi $(24 \mathrm{~nm}) /$ rubrene $(0.2 \mathrm{~nm}) /$ DPVBi $(6 \mathrm{~nm}) / 4,7-$ diphenyl-1,10-phenanthroline (BPhen):cesium carbonate $\left(\mathrm{Cs}_{2} \mathrm{Co}_{3}\right)(10 \mathrm{~nm}) / \mathrm{Mg}: \mathrm{Ag}(6 \mathrm{~nm}) / \mathrm{Al}(120 \mathrm{~nm})$ white organic lightemitting diode (WOLED) with high color purity and stability was fabricated. The function of the multiple-ultrathin material (MUTM), such as rubrene, is as the yellow light-emitting layer and trapping layer. The results show that the MUTM has excellent carrier capture effect, resulting in high color stability of the device at different applied voltages. The Commission Internationale De L'Eclairage (CIE) coordinate of this device at 3-7 V shows a few displacements and a very slight variation of $( \pm 0.01, \pm 0.01)$. The maximum brightness of 9986 $\mathrm{cd} / \mathrm{m}^{2}$ and CIE coordinates of $(0.346,0.339)$ are obtained at $7 \mathrm{~V}$. The enhanced performance of the device may result from the direct charge trapping in MUTM, and it can be found in the electroluminescence (EL) process.

\section{Introduction}

Recently, organic light-emitting diodes (OLEDs) have attracted considerable attention as new full-color displays and solid-state lighting technology. The OLEDs' superior characteristics such as high brightness, fast response time, wide viewing angle, and low operating voltage give it great potential for commercialization, such as a sensing technique or device. ${ }^{(1-3)}$

\footnotetext{
*Corresponding author: e-mail: chenwr@nfu.edu.tw

** Corresponding author: e-mail: chien@nuk.edu.tw https://doi.org/10.18494/SAM.2019.1995
} 
Furthermore, the white organic light-emitting diode (WOLED) lighting technology has been extensively studied. There are several methods of obtaining WOLEDs, for example, using a multilayer stack of three primary colors (red, green, and blue), two complementary colors (blue and yellow), and two or three colors of a dye doped into a single host material. ${ }^{(4-7)}$ In the doping codeposition, accurately controlling the evaporation rate and the concentration of the two or more materials is very difficult, resulting in its deposition and reproducibility becoming poor. However, a nondoping technique can accurately control these factors to avoid these problems during the fabrication. Recently, Tsuji et al. and Xie et al. have reported nondopedtype WOLEDs with the single-ultrathin layer (SUTL) structure, which is a simple device structure with good reproducibility, making them very suitable for low-cost lighting or sensor applications and conducive to commercialization. ${ }^{(8,9)}$

In this work, we employ a nondoping method to fabricate WOLEDs with a multiple-ultrathin material (MUTM) structure. The material 5,6,11,12-tetraphenylnaphthacene (rubrene) was used as yellow light sources in the MUTM structure. Rubrene is a very common nanomaterial, which has good light-emitting efficiency, high color saturation, good stability, and good capture efficiency of charge carriers. In addition, the photoluminescence (PL) quantum efficiency of rubrene can be close to $100 \% .^{(10-13)}$ The material 4,40-bis (2,20-diphenyl vinyl)-1,10-biphenyl (DPVBi) is a well-known blue fluorescent material. It is usually considered as the main emitting layer (EML) owing to the high efficiency of blue light. Moreover, DPVBi is not only emitting but also transferring the incomplete energy from DPVBi to the rubrene. So far, studies of fluorescence WOLED based on the nondoped MUTM are rare. Therefore, we introduced a simple process for the nondoped WOLEDs with a MUTM structure. Meanwhile, the factors affecting the improvement of WOLED performance were investigated in detail. Moreover, the mechanism of the effects of the MUTM structure on the electroluminescence (EL) and optimal color stability of WOLEDs is also presented.

\section{Experimental Details}

Indium tin oxide (ITO)-coated glass substrates with a sheet resistance of approximately $12 \Omega / \square$ were consecutively cleaned in an ultrasonic bath containing detergent water, acetone, ethanol, and deionized (DI) water for 20 min each, then dried with a nitrogen $\left(\mathrm{N}_{2}\right)$ flow. All organic nanomaterials were deposited by high-vacuum $\left(10^{-6}\right.$ Torr $)$ thermal evaporation. The thermal deposition rates for organic nanomaterials, inorganic materials, and Al were about 0.1, 1 , and $10 \AA / \mathrm{s}$, respectively. The evaporation rate and thickness of the thin films were monitored using a quartz crystal oscillator system (Sigma, SID-142). The structures of the devices are as follows:

A. ITO/molybdenum trioxide $\left(\mathrm{MoO}_{3} ; 15 \mathrm{~nm}\right) / \mathrm{N}, \mathrm{N} 0-$ bis-(1-naphthyl)-N,N0-biphenyl-1,10biphenyl-4,40-diamine (NPB; $40 \mathrm{~nm}$ )/DPVBi (10 nm)/rubrene (0.2 nm)/DPVBi (30 nm)/4,7diphenyl-1,10-phenanthroline: cesium carbonate (BPhen: $\left.\mathrm{Cs}_{2} \mathrm{Co}_{3}=4: 1 ; 10 \mathrm{~nm}\right) / \mathrm{Mg}: \mathrm{Ag}(6$ $\mathrm{nm}) /$ Aluminum $(\mathrm{Al} ; 120 \mathrm{~nm})$

B. ITO/ $\mathrm{MoO}_{3}(15 \mathrm{~nm}) / \mathrm{NPB}(40 \mathrm{~nm}) / \operatorname{DPVBi}(34 \mathrm{~nm}) /$ rubrene $(0.2 \mathrm{~nm}) / \operatorname{DPVBi}(6 \mathrm{~nm}) /$ BPhen: $\mathrm{Cs}_{2} \mathrm{Co}_{3}=4: 1(10 \mathrm{~nm}) / \mathrm{Mg}: \mathrm{Ag}(6 \mathrm{~nm}) / \mathrm{Al}(120 \mathrm{~nm})$ 
C. $\mathrm{ITO} / \mathrm{MoO}_{3}(15 \mathrm{~nm}) / \mathrm{NPB}(40 \mathrm{~nm}) / \mathrm{DPVBi}(10 \mathrm{~nm}) /$ rubrene $(0.2 \mathrm{~nm}) / \mathrm{DPVBi}(24 \mathrm{~nm}) /$ rubrene $(0.2$

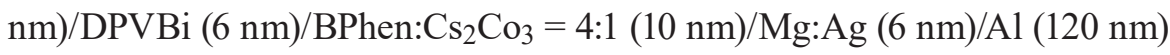

In these devices, $\mathrm{MoO}_{3}$ and NPB are used as the hole-injecting layer and hole-transporting layer, respectively. The DPVBi acts as the blue-EML. The ultrathin rubrene (UTR) was selected as the yello-EML. The Bphen: $\mathrm{Cs}_{2} \mathrm{Co}_{3}$ is used as the electron-transporting layer and hole-blocking layer. The chemical structures of the organic nanomaterials and the structures of the device are shown in Fig. 1. The active area of the device was $0.4 \mathrm{~cm}^{2}$. While measuring the properties of the device, voltage was applied using a Keithley 2400 programmable voltagecurrent source (Keithley SourceMeter 2400; USA). EL spectra and CIE coordination of the devices were measured using PR655 spectra scan spectrometer (Kollmorgen Instrument PR655; USA). All measurements were carried out at room temperature in air without encapsulating the devices.

\section{Results and Discussion}

In this study, the WOLED that consists of the blue and yellow emission layers was fabricated. The UTR layer was inserted in the light-EML of DPVBi to form the structure of DPVBi $(10 \mathrm{~nm}$ EML1)/rubrene $(0.2 \mathrm{~nm}) / \mathrm{DPVBi}(30 \mathrm{~nm}$ EML2) for device A. The yellow emission of device A was caused by the UTR layer. Figure 2(a) shows the EL spectra of device A at the applied voltage of 3-7 V. The peak wavelengths of the DPVBi layer and rubrene layer were 436 and $556 \mathrm{~nm}$, respectively. ${ }^{(14,15)}$ The intensity of the blue emission was higher than that of the yellow emission, and the wavelength of EL spectra for the blue and yellow emissions did not shift as the voltage increased.

In addition, the CIE coordinates of device $A$ at the applied voltage of 3-7 V are shown in Fig 2(b). It was found that the CIE coordinates of device A changed from $(0.268,0.299)$ at $3 \mathrm{~V}$ to $(0.249,0.259)$ at $7 \mathrm{~V}$. By comparing with the CIE coordinates $(0.330,0.330)$ of standard white light, the error value of the CIE coordinates was about $(-0.081,-0.071)$ at $7 \mathrm{~V}$, i.e., the shift

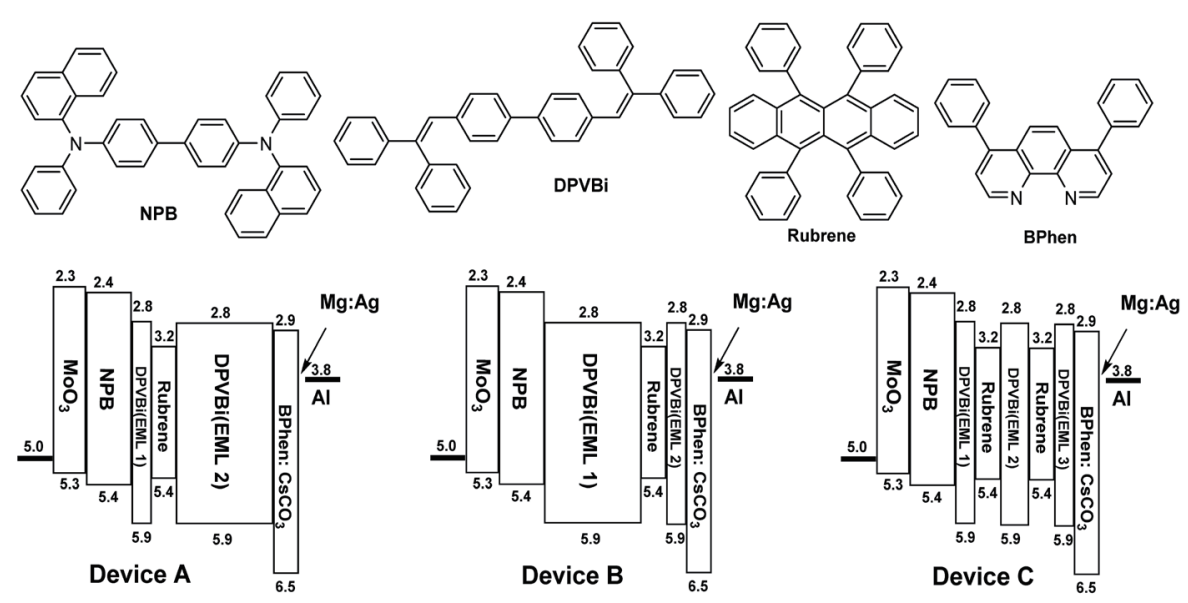

Fig. 1. Chemical structure of organic MUTM and energy level diagram of the WOLED. 


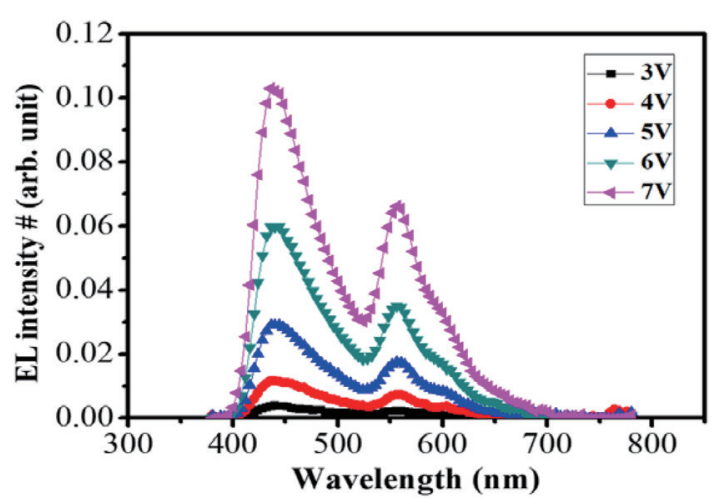

(a)

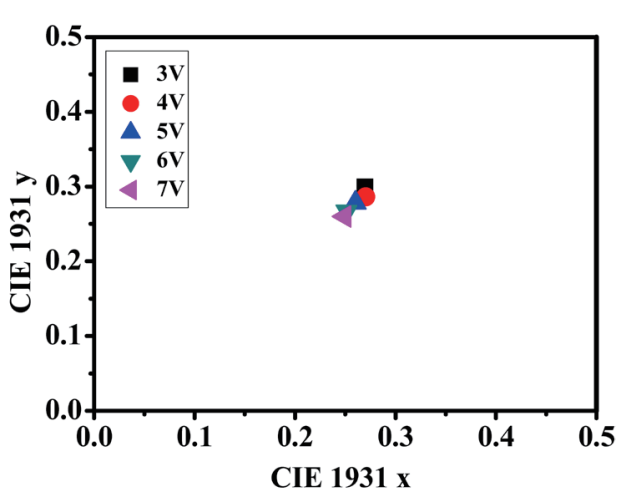

(b)

Fig. 2. (Color online) (a) EL spectra of device A with 3-7 V applied voltage. (b) CIE coordinates of device A with 3-7 V applied voltage.

of the CIE coordinates is about $(-7.4,-13.4 \%)$ when the voltage of $3-7 \mathrm{~V}$ was applied. That is, the CIE coordinates of device A were unstable. As for the EL phenomenon at the applied voltage of 3-5 V, some of the holes that were injected from the anode via the rubrene layer into the EML2 can be directly trapped by the UTR layer, which may be due to the effective hole trapping of rubrene molecules. The UTR layer has excellent charge carrier trapping property, but it is unable to trap many of the injected holes. On the other hand, the electron mobility in BPhen: $\mathrm{Cs}_{2} \mathrm{Co}_{3}$ of $3.89 \times 10^{-4} \mathrm{~cm}^{2} / \mathrm{Vs}$ was less than the hole mobility of $5.5 \times 10^{-4} \mathrm{~cm}^{2} / \mathrm{Vs}$ in NPB, so many electrons from the electrode inject only into EML2, i.e., the electrons recombine with holes in the EML2 close cathode. Therefore, the electrons were difficult to be directly trapped by the UTR layer at 3-5 V. As a result, the great majority of electrons and holes would meet in EML2. Similarly, for the voltage of 6-7 V, more charge carriers were injected into the rubrene layer and EML2, so the amount of recombination for electrons and holes also relatively increased in the rubrene layer and EML2, resulting in an enhancement in the intensity of peaks, as shown in Fig. 2(a). However, the UTR layer in device A cannot trap enough electrons and holes to generate excitons. Therefore, the optimum complementary color of the blue and yellow emission intensities was not achieved.

According to the results obtained above, the best recombination zone might be in the EML2 of device A. For device B, the UTR layer inserted in and close to the cathode to form the structure of DPVBi (34 nm EML1)/rubrene $(0.2 \mathrm{~nm}) / \mathrm{DPVBi}(6 \mathrm{~nm}$ EML2) was fabricated. However, by comparing Figs. 2(a) and 3(a), it is found that the yellow emission intensity of device B was stronger than that of device A because the position of the UTR layer is changed. Although the UTR layer was inserted into the best recombination zone and has an excellent charge carrier trapping effect, the blue and yellow emissions did not reach the best complementary color.

As mentioned above, the recombination zone might be in EML2 of device A. Thus, the UTR layer was inserted into a better recombination zone near the EML2/BPhen: $\mathrm{Cs}_{2} \mathrm{Co}_{3}$ interface of device B. The difference between the lowest unoccupied molecular orbital (LUMO) energy of rubrene $(-3.2 \mathrm{eV})$ and that of DPVBi $(-2.8 \mathrm{eV})$ is $0.4 \mathrm{eV}$. Similarly, the difference 
between the highest occupied molecular orbital (HOMO) of rubrene $(-5.4 \mathrm{eV})$ and that of DPVBi $(-5.9 \mathrm{eV})$ is $0.5 \mathrm{eV}^{(16,17)}$ It is expected that electrons and holes can be trapped in the UTR layer as well as accumulated in the rubrene/EML2 interface, leading to an enhancement in the intensity of yellow emission. Figure 3 shows the EL spectra and CIE coordinates of device B. With increasing applied voltage, most of the electrons and holes can be directly trapped and recombined to form excitons in the UTR layer. Moreover, the relative intensity of the yellow emission is higher than that of the blue emission, i.e., the exciton number of the UTR layer is more than that of the DPVBi layer. In Fig. 3(a), the intensities of both yellow and blue emissions become large, but the ratio of the enhancement is larger in the yellow emission than in the blue emission. Thus, the CIE coordinate gradually shifts toward an orientation of white color. For example, when the applied voltages are 3 and $4 \mathrm{~V}$, the locations of the CIE coordinates are at $(0.462$, $0.481)$ and $(0.425,0.437)$, respectively. By comparing with the CIE coordinates of standard white light, the error value of the CIE coordinates was gradually reduced. It is confirmed that the better recombination zone of device B is in the UTR/EML2 interface.

With the applied voltage increased to $5 \mathrm{~V}$, the recombination zone of electrons and holes gradually shifts toward the UTR/EML2 interface; the corresponding CIE coordinate is $(0.391$, 0.405). We thus conjecture that few excitons are generated in the EML2, resulting in an enhancement in the blue emission. On the other hand, more electrons and holes were trapped in the UTR, i.e., more excitons are generated in the UTR, leading to an enhanced yellow emission. At the voltage of $6-7 \mathrm{~V}$, the CIE coordinates shift again from $(0.368,0.385)$ to $(0.348,0.365)$, and gradually shift toward the CIE coordinates of standard white light. When a high voltage is applied, the concentrations of electrons and holes increase, which significantly influences the zone of the exciton generation. The movements of the electrons and holes are similar toward opposite electrodes. Thus, the zone of the exciton generation in device B becomes as broad as the EML1/UTR and UTR/EML2 interfaces. This implies that the location of UTR not only improves the color shift of the CIE coordinates but also enhances the carrier recombination rate. By comparing with the CIE coordinates of standard white light, the error value of the CIE coordinates was about $(0.018,0.035)$ at $7 \mathrm{~V}$, i.e., the shift of the CIE coordinates is about $(-24.7 \%$, $-24.1 \%$ ) when the voltage of 3-7 V was applied. With the results obtained above, the CIE

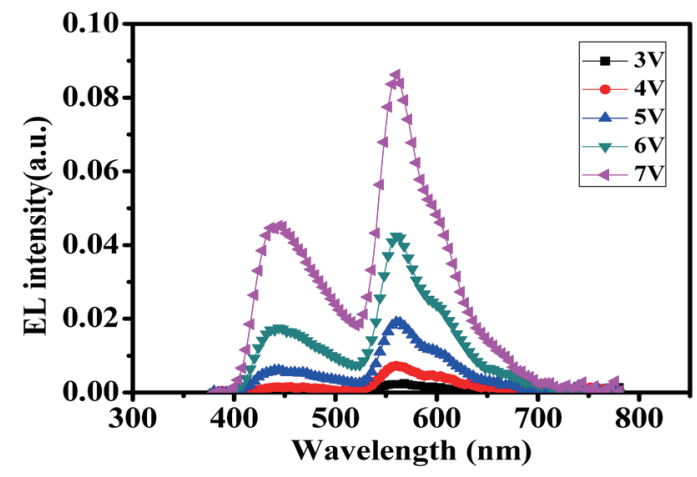

(a)

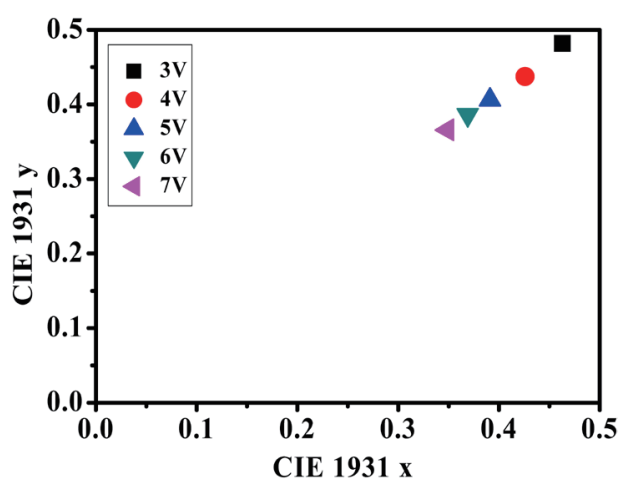

(b)

Fig. 3. (Color online) (a) EL spectra of device B with 3-7 V applied voltage. (b) CIE coordinates of device B with 3-7 $\mathrm{V}$ applied voltage. 
coordinates of device B are near the CIE coordinates of standard white light with the UTR layer inserted into EML2, but the CIE coordinates of device B are still unstable when the voltage of 3-7 V was applied.

Figure 4 shows the EL spectra and CIE coordinates of device $\mathrm{C}$, ITO/ $\mathrm{MoO}_{3} / \mathrm{NPB} / \mathrm{DPVBi} /$ rubrene/DPVBi/rubrene/DPVBi/BPhen: $\mathrm{Cs}_{2} \mathrm{Co}_{3} / \mathrm{Mg}: \mathrm{Ag} / \mathrm{Al}$, when the voltage of $3-7 \mathrm{~V}$ was applied. It is found that there is a balance or complementary color in blue and yellow emissions, as shown in Fig. 4(a). Besides, at the applied voltage of $5 \mathrm{~V}$, a pure white emission with CIE coordinates of $(0.332,0.332)$ is observed. When the voltage continued to increase to 6 and $7 \mathrm{~V}$, the CIE coordinates were respectively $(0.342,0332)$ and $(0.345,0.338)$ and showed little change. This is due to the fact that the ratio of blue to yellow emission intensities from EL spectra of Fig. 4(a) is almost the same and about one. Furthermore, this improvement in chromaticity can be attributed to the MUTM structure in the emission layer, resulting in a balance in the relative intensities of the blue and yellow emissions. Thus, by introducing an MUTM structure in the emission layer, the WOLED has more stable spectra characteristics than devices A and B with the increase in bias voltage. This is because the injection of holes and electrons was enhanced by the MUTM structure and that the MUTM structure can enhance charge carrier trapping in the EML1/rubrene, EML2/rubrene, and rubrene/EML3 interfaces. Then, the carrier injection was enhanced because electric fields were induced by the accumulated carrier at the MUTM. This result is similar to the result discussed in other papers. ${ }^{(18-21)}$ In other words, the excitons formed from electrons and holes can widely distribute in the entire emission layer. As expected, the excitons of rubrene and DPVBi layers will increase and reach a balance, indicating that the MUTM can enhance the color stability of CIE coordinates and the chromaticity of pure white emission. However, the difference in the CIE coordinates when the voltage of 3-7 V was applied is almost zero, which is advantageous for lighting application.

The current density-voltage-luminance characteristics of devices A-C are shown in Fig. 5. The maximum current densities of devices $\mathrm{A}, \mathrm{B}$, and $\mathrm{C}$ are 204,298 , and $356 \mathrm{~mA} / \mathrm{cm}^{2}$ at 7 $\mathrm{V}$, respectively. It can be seen that device $\mathrm{C}$ shows a larger current density than the other two devices at the same voltage. This is the reason why it has a larger luminance. The maximum luminances of devices A-C are 6013, 7769, and $9986 \mathrm{~cd} / \mathrm{m}^{2}$, respectively. It can be explained

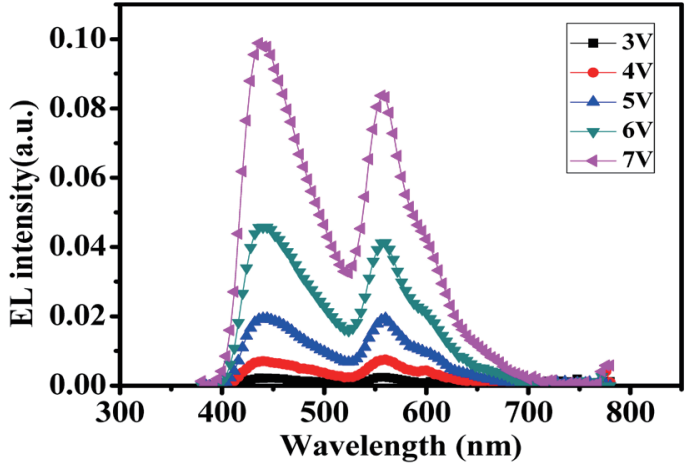

(a)

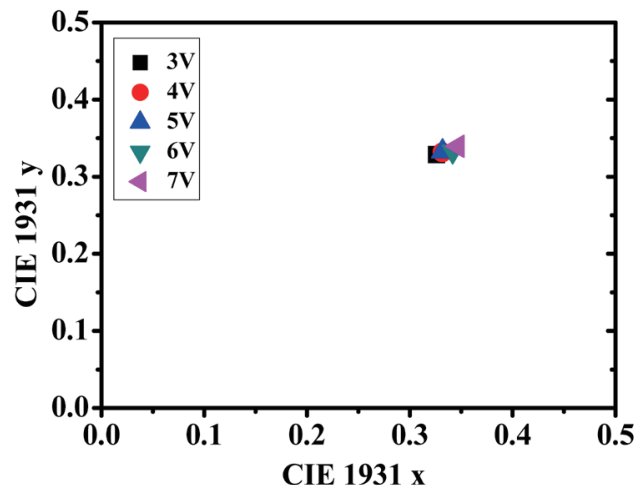

(b)

Fig. 4. (Color online) (a) EL spectra of device $\mathrm{C}$ with 3-7 V applied voltage. (b) CIE coordinates of device $\mathrm{C}$ with 3-7 V applied voltage. 
that the holes were accumulated at the rubrene/EML3 interface and that the electrons were accumulated at the BPhen: $\mathrm{Cs}_{2} \mathrm{Co}_{3}$ layer, indicating that the electric field can be formed in EML3. The electric field is favorable for electron injection into the emission layer. However, it is expected that the holes were trapped and confined in the rubrene/EML3 interface and electron injection was thus enhanced, resulting in an improved carrier balance. Moreover, when the MUTM structure was inserted into the emission layer, the current density increase in device $\mathrm{C}$ exceeds those in the other devices. The phenomenon can be attributed to the effect of the Coulomb attractive force (CAF) that is due to the presence of the trapped and accumulated holes in the emission layer. ${ }^{(2)}$ That is, more accumulated holes in the HOMO level of DPVBi/ rubrene/DPVBi potential well will generate the stronger CAF for electrons, resulting in a better injection of electrons from the electron transport layer to the EML. Thus, the current density increase depends on the position and number of the ultrathin layer, i.e., the formation of MUTM.

Figure 6 shows the current efficiency and power efficiency of the device with and without MUTM. In Fig. 6(a), the current efficiency of device $C$ with MUTM is good and exceeds that without MUTM. At the applied voltage of $3 \mathrm{~V}$, devices $\mathrm{A}, \mathrm{B}$, and $\mathrm{C}$ show the maximum current efficiencies of 3.02, 4.02, and $4.6 \mathrm{~cd} / \mathrm{A}$, respectively. These values indicate that the recombination rate of electron-hole pairs in device $\mathrm{C}$ exceeds those in the other devices. In Fig.

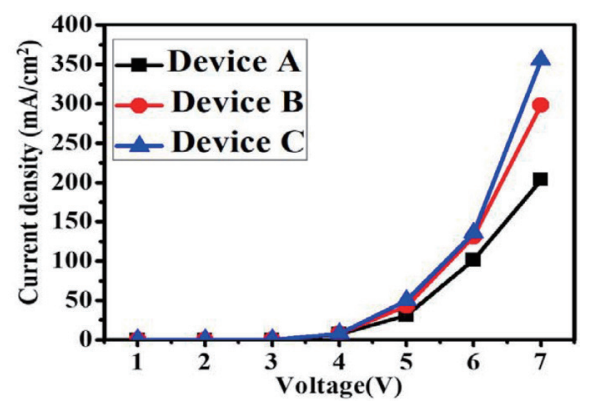

(a)

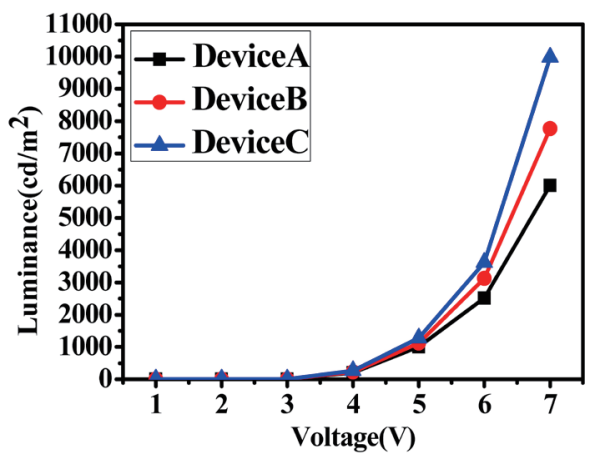

(b)

Fig. 5. (Color online) (a) Current density versus bias voltage $(J-V)$ characteristics of devices A-C. (b) Luminance versus bias voltage $(L-V)$ characteristics of devices A-C.

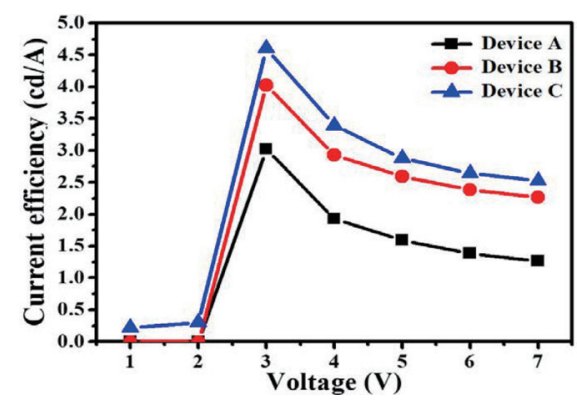

(a)

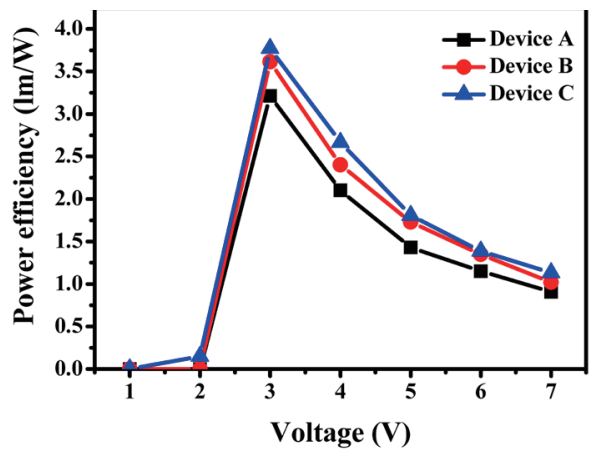

(b)

Fig. 6. (Color online) (a) Current efficiency versus bias voltage characteristics of devices A-C. (b) Power efficiency versus bias voltage characteristics of devices A-C. 
6(b), the maximum power efficiencies of devices $\mathrm{A}-\mathrm{C}$ are $3.2,3.6$, and $3.7 \mathrm{~lm} / \mathrm{W}$, respectively. It can be seen that device $\mathrm{C}$ with the MUTM structure has a high power efficiency than the other two devices. As is known, the power efficiency depends on the carrier injection, while the current efficiency depends on not only the carrier injection, but also the carrier balance. In contrast, an unbalanced injection of electron-hole pairs in the EML with excess carrier injection reduces the efficiency owing to the effect of carrier quenching. Thus, the high current efficiency and power efficiency of the device can be achieved at an efficient carrier balance. However, the MUTM structure with the high carrier trapping efficiency leads to the formation of a stronger CAF, which enhances the efficiency of devices.

\section{Conclusions}

In summary, white light emission was achieved by inserting the MUTM structure in the emission layer. The carrier trapping mechanism of the MUTM structure can more effectively confine electrons and holes in the emission layer. Consequently, the excitons of rubrene and DPVBi layers will increase and reach a balance; i.e., there is a balance or complementary color in blue and yellow emissions, resulting in the good color stability characteristics of WOLED at different applied voltages. Pure white emission CIE coordinates of $(0.332,0.332)$ can be obtained and show a small change at the applied voltage of 3-7 V. It is demonstrated that EL emission is less dependent on the applied voltage. Furthermore, devices with an MUTM, which will be applied to the sensing technique, can increase the carrier-trapping probability to improve carrier recombination, current density, and luminance intensity.

\section{Acknowledgments}

We thank the Research Center for Electro-Optical and Semiconductor, Southern Taiwan University of Technology in Taiwan for the fabrication and measurements of WOLED.

\section{References}

1 C. W. Tang and S. A. Vanslyke: Appl. Phys. Lett. 51 (1987) 913.

2 C. W. Tang, S. A. Vanslyke, and C. H. Chen: J. Appl. Phys. 65 (1989) 3610.

3 C. J. Huang, C. C. Kang, T. C. Lee, W. R. Chen, and T. H. Meen: J. Lumin. 129 (2009) 1292.

4 J. Kido, H. Shionoya, and K. Nagai: Appl. Phys. Lett. 67 (1995) 2281.

5 K. Okumoto, H. Kanno, Y. Hamada, H. Takahashi, and K. Shibata: Appl. Phys. Lett. 89 (2006) 013502.

6 G. Li and J. Shinar: Appl. Phys. Lett. 83 (2003) 5359.

7 K. T. Kamtekar, A. P. Monkman, and M. R. Bryce: Adv. Mater. 22 (2010) 572.

8 T. Tsuji, S. Naka, H. Okada, and H. Onnagawa: Appl. Phys. Lett. 81 (2002) 3329.

9 W. F. Xie, Z. J. Wu, S. Y. Liu, and S. T. Lee: J. Phys. D: Appl. Phys. 36 (2003) 2331.

10 H. Mattoussi, H. Murata, C. D. Merritt, Y. lizumi, J. Kido, and Z. H. Kafafi: J. Appl. Phys. 86 (1999) 2642.

11 Y. M. Wang, T. Feng, X. Zheng, Y. B. Hou, S. Y. Yang, and X. R. Xu: Mater. Chem. Phys. 92 (2005) 291.

12 M. Matsumura and T. Furukawa: Jpn. J. Appl. Phys. 40 (2001) 3211.

13 L. Tianle, L. Xiao, L. Wenlian, B. Chu, Z. Su, H. Lia, Z. Hu, and Z. Zhang: Thin Solid Films 517 (2009) 4629.

14 C. J. Huang, T. H. Meen, K. C. Liao, and Y. K. Su: J. Phys. Chem. Solids 70 (2009) 765.

15 Q. Xue, S. Zhang, G. Xie, Z. Zhang, L. Zhao, Y. Luo, P. Chen, Y. Zhao, and S. Liu: Solid-State Electron. 57 (2011) 35.

16 D. Xu, X. Li, H. Ju, Y. Zhu, and Z. Deng: Displays 32 (2011) 92. 
17 X. Y. Zheng, W. Q. Zhu, Y. Z. Wu, X. Y. Jiang, R. G. Sun, Z. L. Zhang, and S. H. Xu: Displays 24 (2003) 121.

18 L. Li, J. Yu, X. Tang, T. Wang, W. Li, and Y. Jiang: J. Lumin. 28 (2008) 1783.

19 X. Tang, J. Yu, L. Lu, L. Zhang, and Y. Jing: Displays 30 (2009) 123.

20 S. H. Yang, B. C. Hong, and S. F. Huang: J. Appl. Phys. 105 (2009) 113105.

21 Z. Ma, J. Yu, L. Li, and Y. Jiang: Displays 33 (2012) 42.

22 Q. Xu, J. Ouyang, and Y. Yang: Appl. Phys. Lett. 23 (2003) 83. 